\title{
The AMoRE project status
}

\author{
Elena Sala* on behalf of the AMoRE collaboration. \\ Institute for Basic Science, Center for Underground Physics \\ E-mail: elenasala@ibs.re.kr
}

The AMoRE (Advanced Mo-based Rare process Experiment) project aims at observing the neutrino-less double beta decay in ${ }^{100}$ Mo exploiting the well established performances of low temperature detectors. Some compelling issues to achieve high experimental sensitivity in rare events physics experiments concern the requirements for an excellent energy resolution and for a strong background suppression, of which an important contribution is given by alpha contaminants.The AMoRE experiment uses $\mathrm{CaMoO}_{4}$ scintillating crystals read by MMCs (Metallic Magnetic Calorimeters) measuring both heat and light signals: the ratio between these provides indeed a powerful tool for particle identification. The performances of these detectors allow not only to discriminate alpha from beta/gamma events but also provide a good energy and time resolution which are characteristics of MMCs. These state-of-the-art performances combined with background suppression are key features in order to improve the experimental sensitivity of this class of experiments. In this contribution we present the status and prospective of the AMoRE pilot experiment, consisting of $1500 \mathrm{~g}$ of enriched $\mathrm{CaMoO}_{4}$ crystals, which is currently set in the 700 $\mathrm{m}$ deep underground laboratory of $\mathrm{Y} 2 \mathrm{~L}$ (South Korea).

Neutrino Oscillation Workshop

4 - 11 September, 2016

Otranto (Lecce, Italy)

\footnotetext{
* Speaker.
} 


\section{Introduction}

The neutrino oscillation experiments have provided the evidence that at least three massive mass eigenstate neutrinos exist [1], but their absolute mass remains undetermined. Besides, oscillation experiments are not sensitive to the Majorana or Dirac nature of neutrinos. The search for neutrino-less double beta decay $(0 v \beta \beta)$ can access the value of the neutrino mass and thus it is a powerful tool to investigate these properties. This decay, never observed so far, occurs if neutrinos are massive Majorana particles. The process consists in a transition of even-even nucleus with atomic number $Z$ decaying to $Z+2$ nucleus with the emission of only two electrons: this implies the violation of the leptonic number by two units and neutrinos to have non vanishing masses, features not included in the Standard Model. As a consequence of the smallness of the neutrino mass the phase space term is strongly suppressed, this results in an expected extremely low decay rate. The sensitivity for $0 v \beta \beta$ experiments depends mainly on the efficiency, the mass of the candidate isotope, the measuring time, the energy resolution and the background. All these parameters should be optimized; a crucial parameter is the background which has to be as low as possible.

The AMoRE (Advanced Molybdenum-based Rare process Experiment) project aims at searching for the $0 v \beta \beta$ decay of ${ }^{100} \mathrm{Mo}$ [2] using $\mathrm{CaMoO}_{4}$ scintillating crystals acting as energy absorber and source of the decay with a high detection efficiency. Amongst the candidate isotope for this decay, ${ }^{100}$ Mo has the advantages of a relatively short half life for the process under study and a $Q$-value of $3034 \mathrm{keV}$, well above the highest environmental ${ }^{208} \mathrm{Tl} \gamma$ quanta, $2615 \mathrm{keV}$. The crystals have been depleted in ${ }^{48} \mathrm{Ca}$ [3] reaching a level lower than $10^{-3} \%$ in order to avoid the background from the $2 v \beta \beta$ decay of this isotope whose $Q$-value is $4271 \mathrm{keV}$. The total mass of the active isotope has been increased by enriching the crystals in ${ }^{100}$ Mo above $96 \%$. The AMoRE experiment utilises MMCs for both light and heat signals with excellent energy resolution in the heat channel. The simultaneous measurement of these signals allows to perform an efficient background suppression thanks to alpha particles discrimination. Thanks to the high $Q_{\beta \beta}$, the characteristics of the crystals and the high detectors performance, the AMoRE project aims to reach a zero background in the region of interest.

\section{Above Ground Measurement}

The performances of the AMoRE detectors have been first tested above ground at temperatures $10 \mathrm{mK}$ - $30 \mathrm{mK}$ using a ${ }^{48 d e p l} \mathrm{Ca}^{100} \mathrm{MoO}_{4}$ crystal of $196 \mathrm{~g}$ with two sensors to measure both light (photon) and heat (phonon) signals. The phonon collector was made of a patterned gold film evaporated on the crystal bottom surface and then thermally connected through annealed gold wires to the MMC device. The MMC is coupled to a SQUID (Superconducting QUantum Interference Device) which converts the change in the magnetic flux into a measurable voltage signal [4]. The light detector, placed on the top of the crystal, consisted in a 2-inch Ge wafer as scintillation light absorber and a gold film working as phonon collector connected to the MMC and the SQUID sensor [5]. The measurement lasted about $600 \mathrm{~h}$ and provided the evaluation of the discrimination power of the described set up. The PSD on the phonon channel considering the mean rise time of the signals shows two distinct groups of events: the main group consists of $\beta / \gamma$ events and muons, the lower band is characterised by $\alpha$ events (fig.1). The evaluated discriminating power is 
about $20 \sigma$ considering the events from the ${ }^{210} \mathrm{Po} \alpha$ decay $\left(Q_{\alpha}=5.41 \mathrm{MeV}\right)$. Since it is possible to measure simultaneously heat and light, the ratio between them can be a further powerful tool of event separation as shown in figure 2; the evaluated discrimination power, with this method, is about $9 \sigma$.

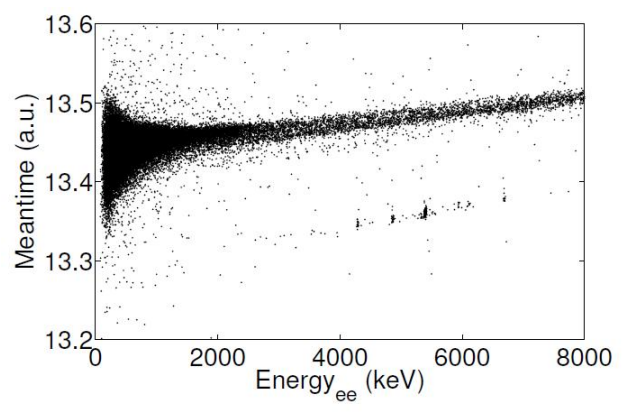

Figure 1: Scatter plot of the Meantime and Pulse height: $\alpha$ events (on the bottom) and $\beta / \gamma$ events are separated.

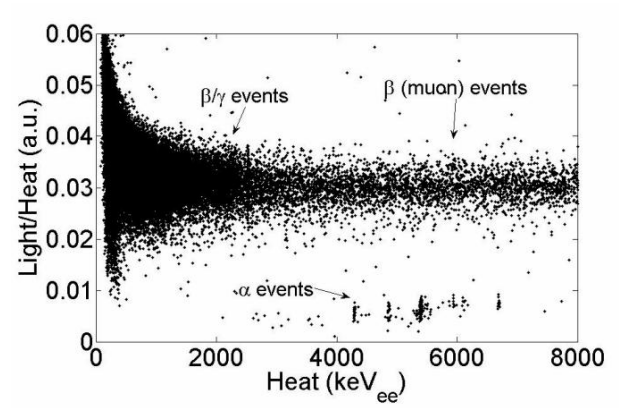

Figure 2: Scatter plot of the Light/Heat ratio: $\alpha$ events (on the bottom) and $\beta / \gamma$ events are well separated.

\section{The AMoRE Pilot}

The AMoRE-pilot experiment [6] consists of a tower of 5 enriched ${ }^{48 d e p l} \mathrm{Ca}^{100} \mathrm{MoO}_{4}$ crystals for a total mass of $1.5 \mathrm{~kg}$ (fig.3). Each crystal is equipped with a phonon detector and a light detector (fig.4) and an additional light detector is placed at the bottom of the tower whose structure is made of NOSV Copper; each module is surrounded by a light reflector to increase the light collection efficiency. To reduce the external background from the cryostat a $100 \mathrm{~mm}$ Lead layer is placed on the top of the tower from the mixing chamber plate. The refrigerator is then further shielded by $150 \mathrm{~mm}$ of low activity Lead. The experimental set up is placed at the Y2L underground laboratory with a maximum coverage of $700 \mathrm{~m}$.

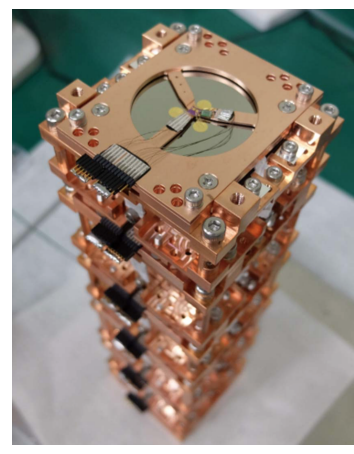

Figure 3: The AMoRE pilot detectors tower.

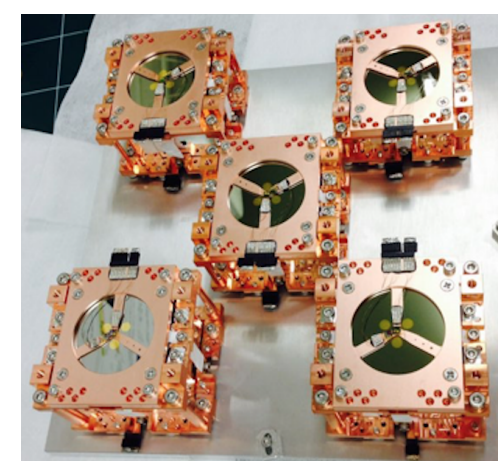

Figure 4: The assembled five crystals of the AMoRE pilot.

The discrimination power of the detector have been degraded in the first $84 \mathrm{~h}$ commissioning run mainly due to the vibration from the pulse tube of the refrigerator. Thanks to the changes in the detector structure (the supporters of the crystals, the light detectors and the wire connections were 


\begin{tabular}{c|c|c} 
Crystal & $1^{\text {st }}$ run & $2^{\text {nd }}$ run \\
\hline SB28 & $36.8 \mathrm{keV}$ & $25.0 \mathrm{keV}$ \\
S35 & none & $16.3 \mathrm{keV}$ \\
SS68 & $52.6 \mathrm{keV}$ & $24.2 \mathrm{keV}$ \\
SE01 & $39.7 \mathrm{keV}$ & $24.6 \mathrm{keV}$ \\
SB29 & $42.6 \mathrm{keV}$ & none
\end{tabular}

Table 1: Comparison of the FWHM on the $2.6 \mathrm{MeV}$ peak during the $1^{\text {st }}$ and the $2^{\text {nd }}$ run .

modified; the phosphor bronze springs, used as holders for the light detectors and the crystals, have been replaced with Teflon) the mechanical vibrations have been reduced and the energy resolution improved. The comparison between the value of the FWHM on the $2.6 \mathrm{MeV}$ peak during the first and the second measurement is shown in table 1. Furthermore a dedicated mass spring damper has been designed to reduce the mechanical vibrations noise caused by the pulse tube refrigerator. This system, which has a total mass of $29 \mathrm{~kg}$, it is currently installed hanging from the mixing chamber via four springs with a $1225 \mathrm{~N} / \mathrm{m}$ spring constant.

\section{Conclusion}

The AMoRE experiment aimed to search the $0 v \beta \beta$ decay in ${ }^{100}$ Mo using scintillating crystals with the MMC technology for the simultaneous measurement of the heat and light signals. To enhance the sensitivity of the experiment the mass of the candidate isotope has been increased by using ${ }^{48 d e p l} \mathrm{Ca}^{100} \mathrm{MoO}_{4}$ enriched crystals. The sensor setup, using MMC devices read out by SQUIDs is used for both phonon and photon channel, has been optimised considering the dimensions of the detectors and the thermal links. A mass spring system has been designed and installed to reduce the vibration noise and a new measurement is now ongoing. The AMoRE experiment has the possibility to reach a zero background level. The expected sensitivity for the pilot stage is $T_{1 / 2} \sim 10^{24}$ years for the half life with a background level in the $0 v \beta \beta$ decay region of $0.01(\mathrm{keV}$ $\mathrm{kg}$ year $)^{-1}$.

\section{References}

[1] J. Beringer, et al., Phys. Rev. D , 86010001 (2012)

[2] H. Bhang, et al., J. Phys. Conf. Ser. 375042023 (2012)

[3] V. V. Alenkov et al., Growth and characterization of isotopically enriched ${ }^{40} \mathrm{Ca}^{100} \mathrm{MoO}_{4}$ single crystals for rare event search experiments, Cryst. Res. Technol., pp. 16, 2011

[4] W. S. Yoon, et al., High energy resolution cryogenic alpha spectrometers using magnetic calorimeters, Journal of Low Temperature Physics, vol. 167, no. 3-4, pp. 280-285, 2012

[5] G. B. Kim, et al., Heat and Light Measurement of $a^{40} \mathrm{Ca}^{100} \mathrm{MoO}_{4}$ Crystal for the AMoRE Double Beta Decay Experiment, IEEE TRANSACTIONS ON NUCLEAR SCIENCE, VOL. 63, NO. 2, APRIL 2016

[6] V.Alenkov et al., Technical Design Report for the AMoRE 0v $\beta \beta$ Decay Search Experiment, [arXiv:1512.05957v1] 18 Dec 2015 